\title{
MDCT Angiography Imaging Presentation of ALCAPA in Adults
}

\author{
Qinyi Dai, Biao Lv, Zhaoqi Zhang \\ Radiology Department, Beijing Anzhen Hospital, Capital Medical University, Beijing, China \\ Email: QY627@yahoo.com.cn
}

Received November 8, 2012; revised December 20, 2012; accepted January 4, 2013

\begin{abstract}
Objective: To make a pictorial presentation of the anomalous origin of left coronary artery arising from the pulmonary artery (ALCAPA) appearances in adults on MDCT angiography. Methods: A retrospective evaluation was performed between 2005 and 2011 by ECG-gated coronary MDCT angiography. Total 8 patients included ( 9 - 51 years, mean age 29 years, 7 female). Image quality was evaluated firstly. Multi planar reformations (MPRs), maximum intensity projections (MIPs) and 3D volume-rendering techniques were used to evaluate image features. In addition, right and left coronary artery orifices were measured for each case. Results: Total 8/70,000 cases diagnosed ALCAPA by MDCT exam, all image qualities were acceptable. As the diagnose key point, all left coronary artery origin were clearly described, and indirect signs such as dilated and tortuous right/left arteries and collateral vessels between them, enlarged left ventricle were also well showed, the dilated degree of both the RCA and the LCA were marked with ages, the diameter of RCA for each case were a mild wider than that of left one. Other combined signs like papillary muscle calcification, bronchial arteries from aorta to the cardiac and coronary artery degeneration change were also included by MDCT angiography findings in a single data acquisition. No combined inter cardiac malformations in our group. Conclusion: ECG-gated MDCT angiography plays an important role as a first-line modality in assessment of ALCAPA.
\end{abstract}

Keywords: MDCT Angiography; Bland-White-Garland Syndrome; Coronary Artery Anomalous; Congenital Heart Disease

\section{Introduction}

Anomalous origin of the left coronary artery arising from the pulmonary artery (ALCAPA) is an extremely rare but serious congenital cardiac abnormality. ALCAPA, also known as Bland-White-Garland syndrome [1], is a rare congenital defect, accounting for $0.25 \%-0.50 \%$ of all congenital heart disease, 1 in 300,000 live births. Although magnetic resonance imaging (MRI) is considered the technique of choice in patients with ALCAPA [2], because of the higher spatial resolution and faster exam time of multi-detector computed tomography (MDCT) angiography improved capabilities for assessment of both intra-cardiac anatomy and coronary arteries in a single data acquisition, this modality plays an important role in clinical practice [3]. The purpose of this article is to make a pictorial presentation of ALCAPA appearances in adults on MDCT angiography.

\section{Materials and methods}

\subsection{Patient Population}

A retrospective evaluation was performed to identify all patients who underwent retrospective electrocardiogra- phically-gated (ECG) coronary MDCT angiography between 2005 and 2011 at our cardiac-vascular specialist hospital. Total 8 patients constitute the study group ranged in age from 9 to 51 years (mean age, 29 years) during nearly 70,000 cases; 7 were female. The exclusion criteria were allergy to iodine containing contrast medium, renal insufficiency (serum creatinine concentration $>120 \mu \mathrm{mol} / \mathrm{L}$ ) and unstable clinical condition. All patients were written the informed consent before examination.

\subsection{MDCT Scanning Protocol}

Patients were performed by 64-slice CT (Aquilion one, Toshiba Medical Systems, Japan; $\mathrm{n}=2$ ) and dual-source CT (DSCT, Somatom Definition, Siemens Healthcare, Forchheim, Germany; $\mathrm{n}=6$ ). A $60-75 \mathrm{~mL}$ bolus of iopamidol $(370 \mathrm{mg} / \mathrm{mL}$; Bayer Schering Pharma) followed by $30 \mathrm{~mL}$ of saline solution was continuously injected into an antecubital vein at a flow rate of $2.5-4.0$ $\mathrm{mL} / \mathrm{s}$. The scanning range covered the entire heart and MDCT angiography was triggered automatically within a region of interest in the ascending aorta, image acquisition was initiated 5 seconds after attenuation reached the 
predefined threshold of $180 \mathrm{Hu}$. Data were acquired in a craniocaudal direction with a detector collimation (64 slice CT/DSCT) of $64 \times 0.5 \mathrm{~mm} / 2 \mathrm{~mm} \times 32 \mathrm{~mm} \times 0.6$ $\mathrm{mm}$, gantry rotation time of $400 \mathrm{~ms} / 330 \mathrm{~ms}$, tube voltage of $100-135 \mathrm{kV} / 80-120 \mathrm{kV}$, and both tube current 350 420 mAs. Images were reconstructed with a section thickness of $0.5 / 0.6 \mathrm{~mm}$, both reconstruction increment of $0.3 \mathrm{~mm}$, and soft-tissue convolution kernel.

In all patients, mid-diastolic reconstructions were first performed at $75 \%$ of the R-R interval. If image quality in this data set was a not optimal, additional reconstruction were performed by multiple phase's reconstructions.

\subsection{MDCT Image Analysis}

All data were transferred to a dedicated workstation (Vitrea 3.8, Vital Images) applying a combination of the original axial images, multi planar reformations (MPRs), maximum intensity projections (MIPs) and 3D volume-rendering techniques (VRTs). Image quality for each case was evaluated on a 3-point scale: 1) good image quality with no or with minor artifacts; 2) moderate image quality with moderate artifacts or blurring but adequate for clinical diagnosis; and 3) poor image quality with severe artifacts that made vessel delineation impossible. Segments with score 3 were not considered for further analysis. The consensus interpretation was performed by an observer who with 10-year cardiac image diagnostic experience. In addition, each case the proximal diameter of the right coronary artery (RCA) and the left coronary artery (LCA) were measured by MPRs images.

\subsection{Statistical Analysis}

Statistical analysis was performed with software SPSS13.0. All variables are resented as mean \pm standard deviation,

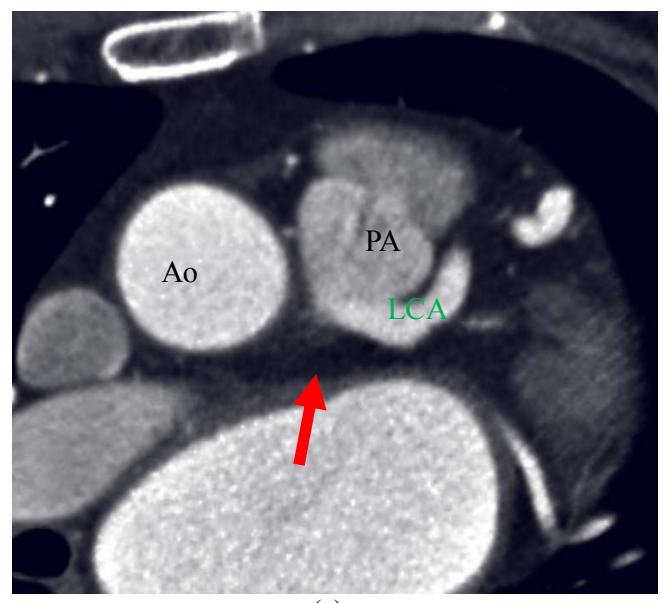

(a) counts, or percentages.

\section{Results}

Eight cases of MDCT angiography were successfully performed and all patients without complications. Average heart rate during the CT scan was from 68 to $90 \mathrm{bpm}$ (average $77 \pm 9 \mathrm{bpm}$ ). All images quality was acceptable, including 6 cases were scale 1 and 2 cases with scale 2 . Clinical symptom were varies from chest not comfortable, chest pain to heart failure and transient syncope history. Besides 1 patient refused operation, other 7 patients underwent surgery successfully and additional valve repair for one of them due to moderate mitral valve regurgitation.

VRTs and MPRs images to be used to describe LCA and RCA origin, all LCA were originated from left-posterior side of main pulmonary arteries (Figure 1(a)), while RCA were arisen from the normal right coronary sinus (Figure 1(b)). The origin diameter of RCA and LCA were 5 - $17 \mathrm{~mm}$ (average $11 \pm 4 \mathrm{~mm}$ ) and $4-15$ $\mathrm{mm}$ (average $9 \pm 4 \mathrm{~mm}$ ) respectively (Figure 2).

Dilated and tortuous left and right arteries and collateral vessels between them were found for every case (Figure 3 and 4); no combined cardiac malformations were detected. All cases were showed enlarged left ventricle and 1 case with marked papillary muscle calcification (Figure 5). Two cases were detected small vascular sup- ply to the cardiac from the aorta (Figure 3). Coronary artery wall thickness with calcification changes were found in 3 cases (Figure 5), but no severe stenosis was detected.

\section{Discussion}

The ALCAPA anomaly may result from (1) abnormal septation of the conotruncus into the aorta and pulmonary

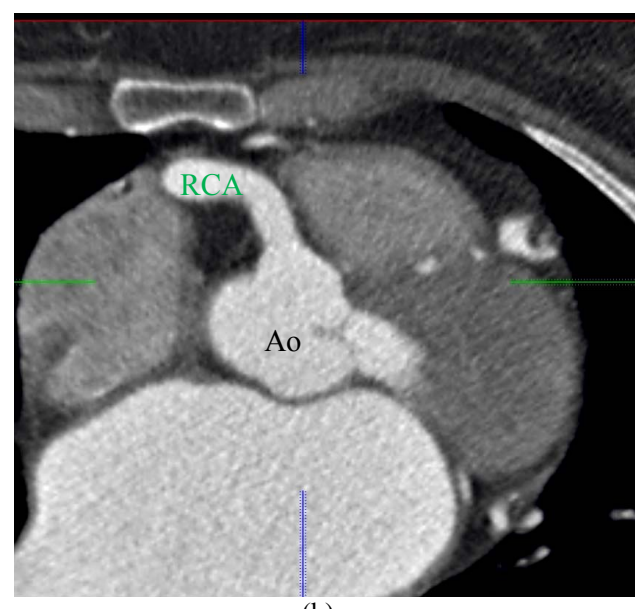

(b)

Figure 1. 28-year-old woman diagnosed ALCAPA by MDCT angiography. MPRs showed (a) The origin of the LCA (arrow) from the left-posterior side of main pulmonary artery (PA) while (b) The dilated RCA arising from the normal site (arrowhead). Measured the orifice of LCA and RCA were $9 \mathrm{~mm}$ and $11 \mathrm{~mm}$ respectively. Ao = aorta. 


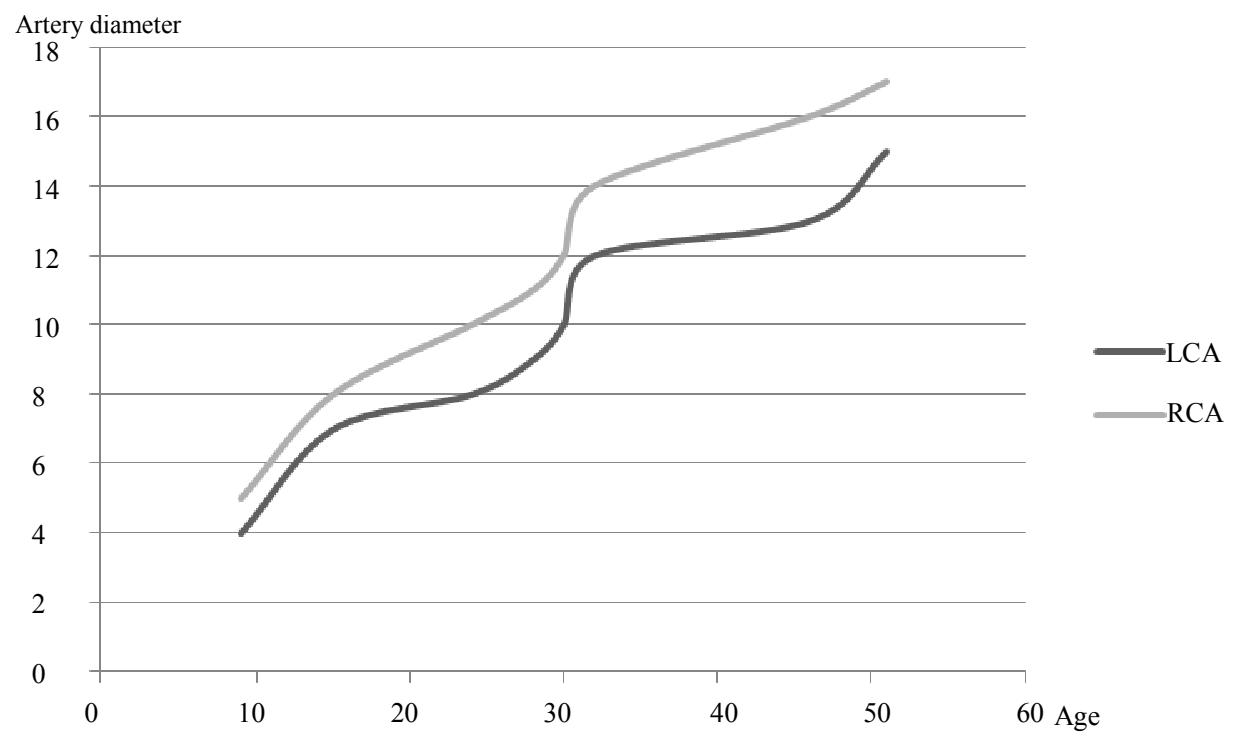

Figure 2. Right coronary artery (RCA) and left coronary artery (LCA) origin diameters (mm) in 8 cases of ALCAPA by MDCT angiography (Age: years).

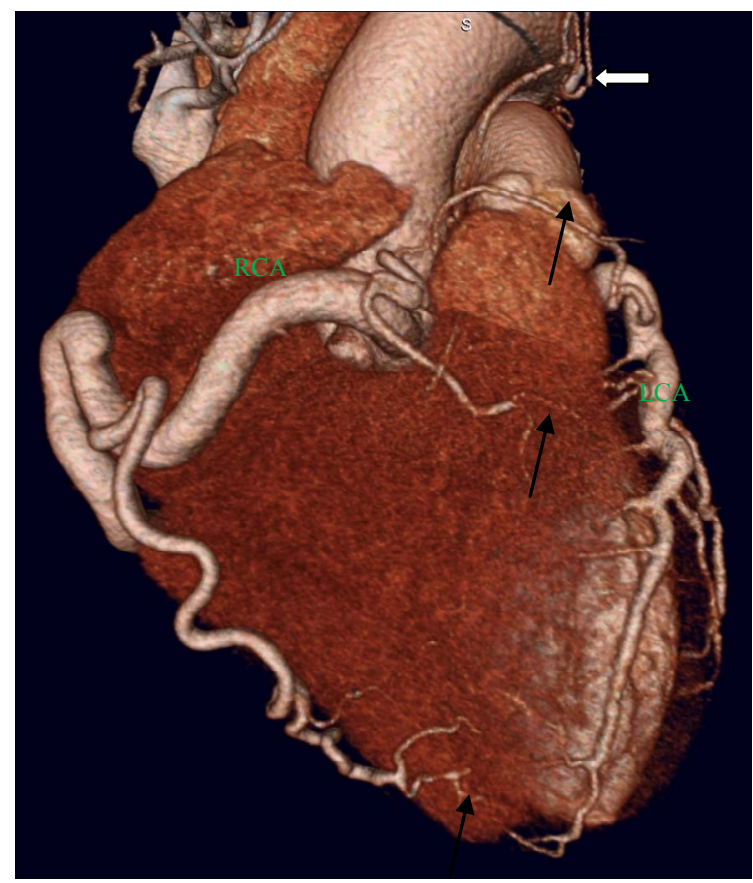

(a)

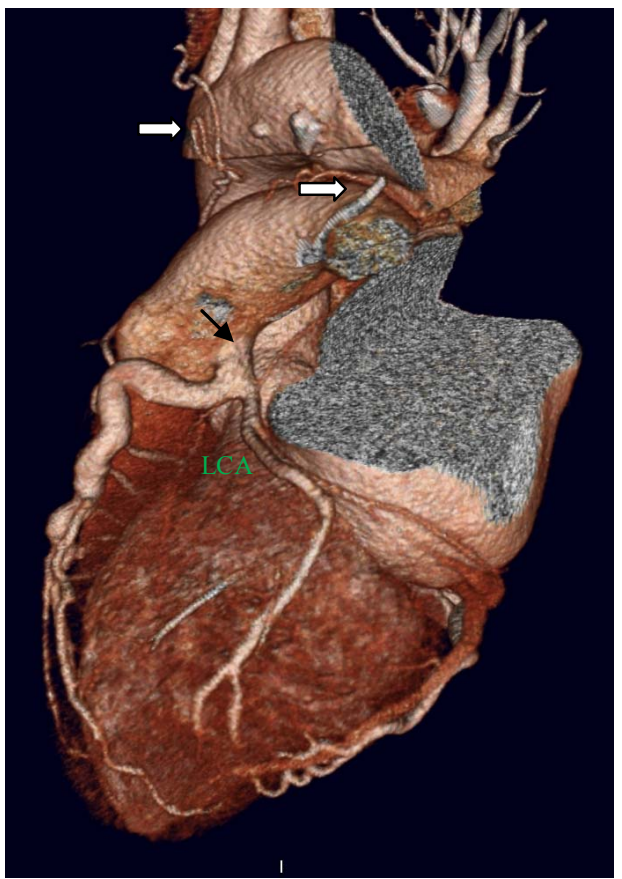

(b)

Figure 3. 24-year-old woman. VRTs shows (A) dilated inter-coronary collateral arteries at the cardiac surface (long arrow), which connect the dilated and tortuous RCA to the LCA. (B) Note the origin of the LCA from the left-posterior side of main pulmonary artery (short arrow) and multiple dilated bronchial arteries arising from the aorta (open arrow). Measured the orifice of LCA and RCA were $8 \mathrm{~mm}$ and $10 \mathrm{~mm}$ respectively.

artery, or from (2) persistence of the pulmonary buds together with involution of the aortic buds that eventually form the coronary arteries. There are two classic types of ALCAPA: the infant type and the adult type. Infants experience myocardial infarction and congestive heart failure and approximately $90 \%$ die within the first year of life. While in adults with well developed significant col- lateral circulation over time from the RCA to the LCA, however, it is still not sufficient to supply the left ventricle, and then myocardial infarction, left ventricular dysfunction, mitral regurgitation or malignant arrhythmias occurred, which can also lead to cardiac death. For adults, risk of sudden death of between $80 \%$ and $90 \%$ at a mean age of 35 [4], old ALCAPA more than 70 years' patients 


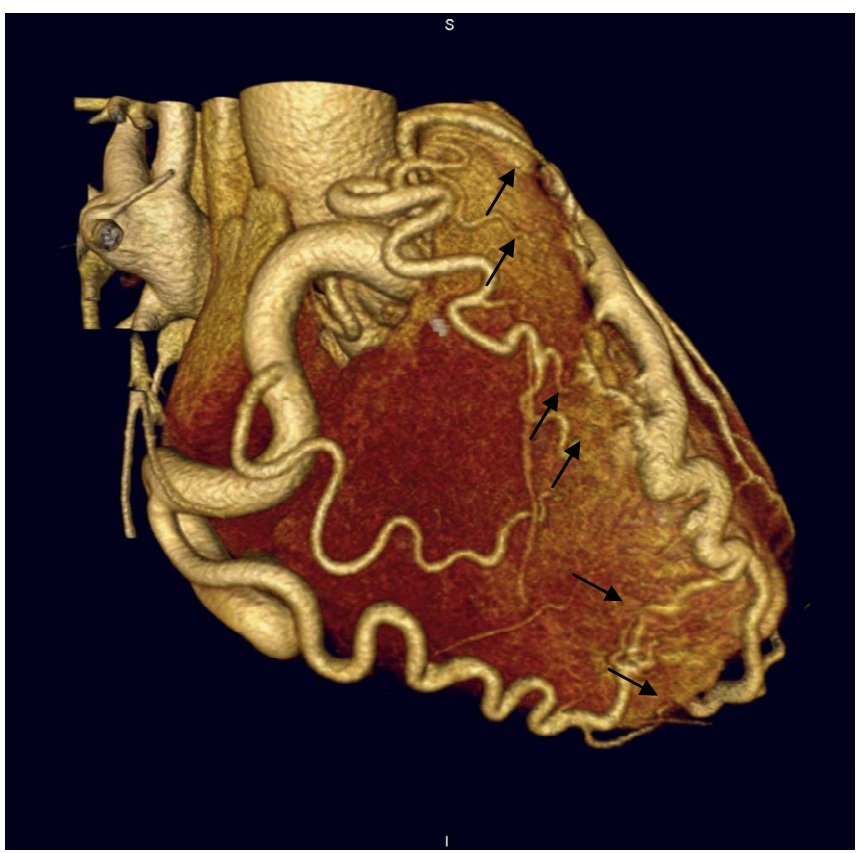

Figure 4. 51-year-old woman. VRT anterior view of RCA with multiple collaterals directed towards the left side of the heart. Note the corkscrew-like appearance of the large ventricular branch of the RCA, compared with Figure 2, the diameter of RCA and collateral vessels along the cardiac surface (arrows) were richer and more obvious for this older patient. Measured the RCA orifice was $17 \mathbf{~ m m}$.
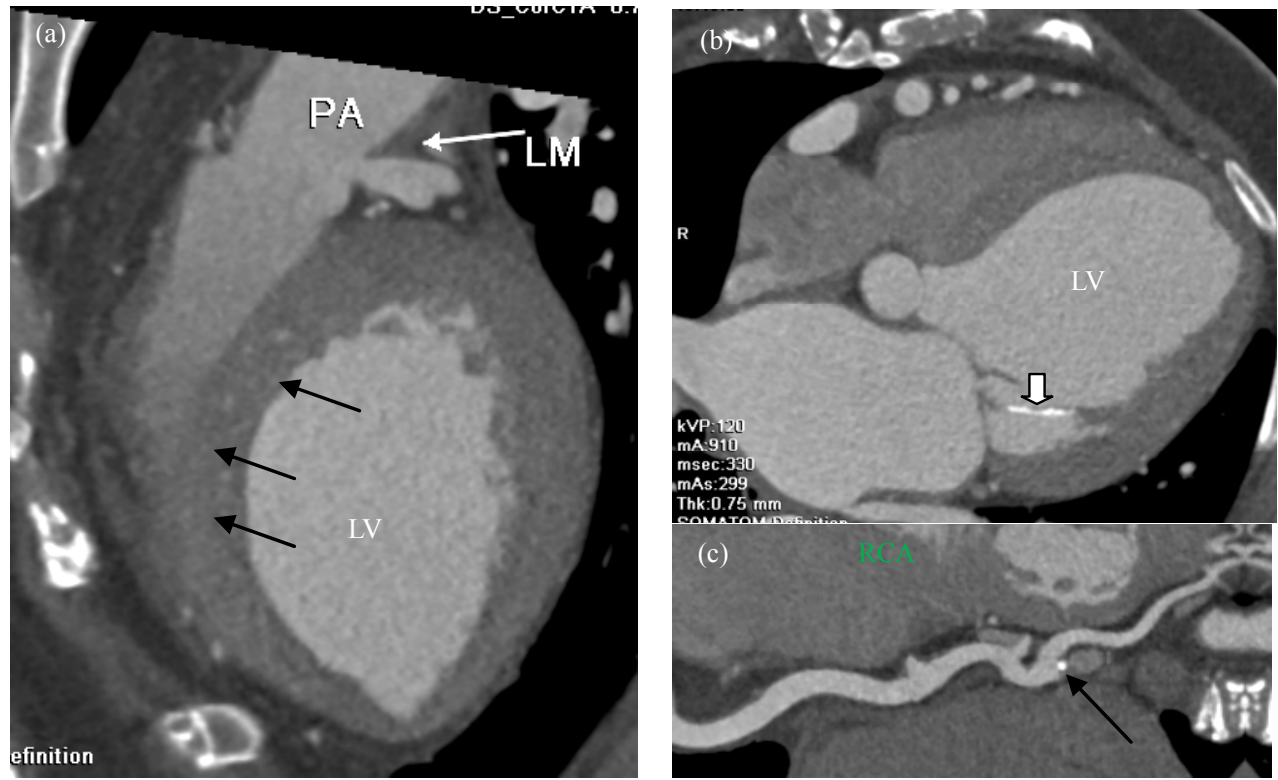

Figure 5. 46-year-old woman. ((a) and (b)) MPRs showed left main artery (LM) arising from the main pulmonary artery (PA), very close to the valvular commissure, thickened myocardial wall and enlarged left ventricle (LV), note the multiple septal collateral vessels (Figure 5(a) arrows) and the remarked papillary muscles calcified (Figure 5(b) open arrow) and dilated RCA. (c) CPR demonstrated spot-like calcified at the distal dilated RCA (arrow).

would be found by case report [5]. Early diagnosis ALCAPA and prompt surgical intervention with the aim of restoring a two-coronary-artery circulatory system have excellent results and lead to gradual myocardial recovery. The restoration of a two-coronary system is the objective of the surgery. Treatment of ALCAPA consists of rec- reation of dual coronary perfusion, either (a) direct reimplantation of the anomalous LCA into the aorta or (b) creation of an intrapulmonary conduit from the left coronary ostia to the aorta (Takeuchi procedure) may be used [6].

In our study, patients average age is 29 years old and 
symptoms were varies from mild chest not comfortable to syncope or heart failure, the lack of serious symptoms besides well-developed collateral vessels, may also been due in part to systemic hypertension, which preserved antegrade blood flow in the RCA [4]. Occurrence is generally no predilection in sex or race, and is not considered an inheritable congenital heart defect. In our study, all 8 patients are Chinese, female predominant and without combined with intra-cardiac disease.

ECG-gated MDCT angiography findings of ALCAPA in adults include direct visualization of the LCA original from the main pulmonary artery, which is the diagnostic key point viewed by MIPs and VRTs, the LCA typically arises from the left-posterior aspect of the main pulmonary artery beyond the pulmonary valve (Figure 1(a)), which were the most common position, however, its position within the sinus is extremely variable, particularly it may be very close to a valvular commissure (Figure 5(a)). Rarely, the anomalous ostium originates from the right posterior sinus or even one of the main pulmonary arteries. To know the exact LCA origin position may help the surgical doctor to determine the optional operation method. In our study, 7 of 8 cases allowed operation successfully, including direct re-implantation $(\mathrm{n}=3)$ and Takeuchi $(n=4)$ surgery. Such images give the surgeons a better understanding of the complex anatomy before repair.

The remarkable secondary imaging finding is the RCA and the LCA dilated and tortuous, and dilated intracoronary collateral arteries are seen along the epicardial surface of the heart by VRTs (Figure 3(a)) or within the interventricular septum by MIPs or MPRs (Figure 5(a)). All cases represent the well-developed collateral pathways between the RCA and the LCA, MDCT images offers excellent spatial resolution, which is required to assess small vessels such as the coronary arteries. In our study, the dilated degree of both the RCA and the LCA marked with ages, the diameter of RCA for each case were a mild wider than that of left one (Table 2, Figure 1 and 3), and collateral vessels along the cardiac surface were richer for older patients (Figure 4). Moreover, the dilated bronchial arteries from the aorta (Figure 3), which act as systemic collateral vessels to the LCA area's blood supply, are best depicted at ECG-gated MDCT angiography because of their small size.

Besides, left ventricular hypertrophy and dilatation result from chronic myocardial ischemia also be seen in MDCT images (Figure 5), may be used to assess left ventricular function. If ischemia of the papillary muscles happened and adjacent myocardium may cause mitral insufficiency, as a finding, papillary muscles calcified can easily be seen at MPRs besides left ventricle enlarged (Figure 5(b)).

Due to the marked dilatation develops over time as blood is rapidly shunted from the RCA into the LCA and then into the low-pressure pulmonary circulation, the degeneration change of the coronary artery could be found in our mean age 29 years ALCAPA group (Figure 5(c)).

2D echocardiography with Doppler color flow mapping in some situations replaces angiography [7], echo is sensitive to determine the mitral valve and left ventricle function, which is also a convenient method used for follow-up. Another technique used to non-invasively image the coronary arteries is MR angiography, MR could accurately depict the origin and proximal course of anomalous coronary arteries [8] and no radiation exposure, even more crucial for MR is the ability to assess myocardial viability, which can be used as an important prognostic factor to consider by the surgical doctor. Conventional coronary angiography still remains as a gold standard for the diagnosis of anomalous coronary arteries; however, it is invasive and limited to a projection two-dimensional view of the complex coronary artery system [9], especially for the retrograde ostium.

ALCAPA should be different with other disease which may cause coronary artery dilated, such as Kawasaki disease, coronary artery fistula or secondary to the atherosclerosis change, the essential feature is to show and determine the LCA origin area.

ECG-gated MDCT angiography plays an important role as a first-line modality in assessment of ALCAPA, and MDCT angiography also would be a valuable evaluated post-operation condition [10]. But our study is limited both by its retrospective nature and a relatively small number of patients. Because of potential drawbacks of MDCT such as radiation exposure and contrast mediumrelated complications, performing MDCT should be limited to patients presenting symptoms suggesting the presence of anomalous coronary arteries.

\section{REFERENCES}

[1] H. Brooks, "Two Cases of an Abnormal Coronary Artery of the Heart Arising from the Pulmonary Artery: With Some Remarks upon the Effect of This Anomaly in Producing Circoid Dilation of the Vessels," Journal of Anatomy and Physiology, Vol. 20, No. 1, 1885, pp. 26-29.

[2] J. C. Post, A. C. van Rossum, J. G. Bronzwaer, C. C. de Cock, M. B. Hofman and J. Valk, "Magnetic Resonance Angiography of Anomalous Coronary Arteries. A New Gold Standard for Delineating the Proximal Course?" Circulation, Vol. 92, No. 11, 1995, pp. 3163-3171. doi:10.1161/01.CIR.92.11.3163

[3] T. Horisaki, T. Yamashita, H. Yokoyama, K. Urasawa and A. Kitabatake, "Three-Dimensional Reconstruction of Computed Tomographic Images of Anomalous Origin of the Left Main Coronary Artery from the Pulmonary Trunk in an Adult," American Journal of Cardiology, Vol. 92, No. 7, 2003, pp. 898-899.

doi:10.1016/S0002-9149(03)00913-5 
[4] C. Frescura, C. Basso, G. Thiene, D. Corrado, T. Pennelli and A. Angelini, "Anomalous Origin of Coronary Arteries and Risk of Sudden Death: A Study Based on an Autopsy Population of Congenital Heart Disease," Human Pathology, Vol. 29, No. 7, 1998, pp. 689-695. doi:10.1016/S0046-8177(98)90277-5

[5] A. Khanna, D. A. Torgian, V. A. Ferrari, R. J. Bross and M. A. Rose, "Anomalous Origin of the Left Coronary Artery from the Pulmonary Artery in Adulthood CT and MRI," American Journal of Roentgenology, Vol. 185, No. 2, 2005, pp. 326-329.

[6] J. Caspi, T. W. Pettitt, C. Sperrazza, T. Mulder and A. Stopa, "Reimplantation of Anomalous Left Coronary Artery from the Pulmonary Artery without Mitral Valve Repair," Annals of Thoracic Surgery, Vol. 84, No. 2, 2007, pp. 619- 623. doi:10.1016/j.athoracsur.2007.03.036

[7] Y. L. Yang, N. C. Nanda, X. F. Wang, M. X. Xie, Lu Q., L. He and X. F. Lu, "Echocardiographic Diagnosis of Anomalous Origin of the Left Coronary Artery from the
Pulmonary Artery," Echocardiography, Vol. 24, No. 4, 2007, pp. 405-411. doi:10.1111/j.1540-8175.2006.00406.x

[8] N. H. Bunce, C. H. Lorenz, J. Keegan, J. Lesser, E. M. Reyes, D. N. Firmin and D. J. Pennell, "Coronary Artery Anomalies: Assessment with Free-Breathing Three-Dimensional Coronary MR Angiography," Radiology, Vol. 227, No. 1, 2003, pp. 201-208. doi:10.1148/radiol.2271020316

[9] I. A. Williams, W. M. Gersony, W. E. Hellenbrand, “Anomalous Right Coronary Artery Arising from the Pulmonary Artery: A Report of 7 Cases and a Review of the Literature," American Heart Journal, Vol. 152, No. 5, 2006, pp. 9-17.

[10] E. Pepa, E. Nguyen, N. Merchant and G. Dennie, "ALCAPA Syndrome: Not Just a Pediatric Disease," Radiographics, Vol. 29, No. 2, 2009, pp. 553-565. doi:10.1148/rg.292085059 